\title{
On the problem of the interpretation of quantum physics
}

\begin{abstract}
A. A. Grib1
Abstract. Current thinking on the interpretation of quantum physics is reviewed, with special detail given to the Copenhagen and Everett many-worlds interpretations.

PACS numbers: 01.65.+g, 01.70.+w, 03.65.-w
\end{abstract}

Methodological Notes.

\section{Contents}

1. Introduction

2. Basic postulates of quantum theory

3. Copenhagen interpretation

4. Everett interpretation

4.1 Discussion topics

5. Conclusion

References

\section{Introduction}

In an earlier paper [1, we made an attempt to show — by reviewing Russian physics textbooks and in the light of recent theoretical developments (Bell inequalities, the Kochen-Specker theorem [2][6]) and the new Bell inequality violation and teleportation experiments of Aspect, Zeilinger, and others [7, 8] - how quantum physics should not be interpreted today, the beginning of the 21st century. We refrained, however, from discussing how it should be interpreted. It is the purpose of this paper to address this issue, presenting some constructive considerations about such an interpretation.

Let us note, to begin with, that microworld phenomena, from elementary particles to molecules, as well as the phenomena of superconductivity and superfluidity, are described so successfully by quantum theory that no one in the physics community has any doubts as to the validity of its mathematical formalism, which has demonstrated such brilliant predictive power. It is attempts

\footnotetext{
${ }^{1}$ A.A. Grib Herzen Russian State Pedagogical University, nab. r. Moiki 48, 191186 St. Petersburg, Russian Federation

E-mail: Andrei_Grib@mail.ru
} 
to interpret this formalism, i.e., to explain it in terms of ordinary language, which has split the physics community. The dissatisfaction with the philosophical implications of this formalism has prompted some physicists to try to change it in some ways: by introducing hidden parameters, by renouncing the superposition principle, or by extending quantum theory by introducing decoherent histories [9]-12]. These alterations have failed, however, to produce a theory that could compete with that taught by textbooks, so interpretations that suggest changing the mathematical formalism of quantum physics will not be considered here. This leaves us with the following three possibilities:

1. Pragmatic interpretation. The rules of quantum theory allow highly accurate predictions to be made about the results of our observations, and this is enough for us. We will simply accept them as given and ask no questions as to why they are as they are and not otherwise.

2. Copenhagen interpretation. This interpretation comes in a number of versions, one of which is item 1. The present paper discusses one relatively recent formulation of the Copenhagen interpretation, put forth by Niels Bohr's son Aage Bohr [13, who summarizes many years of the interpretation debate on quantum mechanics in Copenhagen in the latter half of the 20th century.

3. Everett many-worlds interpretation. First proposed in 1957 by H Everett [14] and propagated by J Wheeler [15], B DeWitt [16] and others, the many-worlds interpretation is used by such cosmologists as S Hawking [17, 18], J Hartle, and A Vilenkin [19] in their attempts to explain the quantum birth of the Universe based on the idea of the Universe's wave function existing in the absence of an observer. In the Russian literature, UFN discussion papers by M B Menskii [20, 21] should be mentioned.

\section{Basic postulates of quantum theory}

Below, we list the main textbook postulates of quantum theory. (Some revision of these postulates in the case of the Everett interpretation leaves the mathematics unchanged.)

(1) The state of a quantum object (for simplicity, the object will be assumed to be a particle) is defined by its wave function $\Psi(x, y, z, t)$, which is a function of Minkowski spacetime coordinates and which is a Hilbert space vector transformable as a unitary representation of the Poincaré group under transformations from this group.

(2) The observed properties of a quantum object are described by self-adjoint operators in a Hilbert space. The set of these operators includes, first of all, the following Poincaré group generators: the Hamiltonian (translation in time), momentum (translation in space), angular momentum and spin (three spatial rotations), and three space coordinate operators as a nonrelativistic limit of 
the three Lorentz rotations. Importantly, as pointed out in review 13, the noncommutativity of and hence the Heisenberg uncertainty principle for - coordinates and momentum follows from the noncommutativity of transformations of spatial translations and Lorentz rotations, which points to a direct relationship between quantum physics and the theory of relativity. Without Minkowski spacetime, quantum physics would be, generally speaking, entirely different from what it is now.

Besides the ones listed above, the set of observables generally includes those without classical analogs: space and time parity (space reflection and time reversal operations from the full Poincaré group), charge parity, and various kinds of charge (electric, baryon, lepton, strange, charm, beauty, height, etc.). Finally, among the observables there are local operators expressible in terms of local quantized fields. For the electromagnetic field and other material fields, the observables are the fields themselves, whereas for electron-positron, baryon-antibaryon and other nonmaterial fields, the observables are bilinear combinations of the field operators.

I. In the absence of measurements, the wave function varies with time in a deterministic way according to the Schrödinger equation

$$
i \hbar \frac{\partial \Psi}{\partial t}=\hat{H} \Psi
$$

and the wave function is transformed through the unitary transformation

$$
\Psi\left(t_{0}\right) \rightarrow \Psi(t)=U\left(t-t_{0}\right) \Psi\left(t_{0}\right)=\exp \left[-i \hat{H}\left(t-t_{0}\right)\right] \Psi\left(t_{0}\right)
$$

In the Heisenberg representation, the wave function does not vary with time; what does vary is observable operators: $\hat{A} \rightarrow U \hat{A} U^{-1}$.

II. Wave packet reduction postulate. When measuring the observable $\hat{A}$ with an instrument, the wave function $\Psi(t)$ collapses into one of the eigenfunctions of operator $\hat{A}$, so that if

$$
\Psi=\sum_{n} c_{n} u_{n}
$$

where

$$
\hat{A} u_{n}=\lambda_{n} u_{n}
$$

then the probability that the instrument measuring property $A$ will provide a reading $\lambda_{n}$ is given by the Born rule:

$$
w_{n}=\left|\left(u_{n}, \Psi\right)\right|^{2}
$$

It is important to note that it is precisely the reduction process [22, 23] which gives rise to randomness, i.e., indeterminism. Into which function $u_{n}$ of the set $\left\{u_{n}\right\}$ with $\left|c_{n}\right|^{2} \neq 0$ the wave function 
$\Psi(t)$ of a quantum object transforms is totally unpredictable. Here is von Neumann's well-known quotation on this point: in quantum mechanics, "dispersions are not due to our lack of knowledge about the state, but to nature itself, which has disregarded the principle of sufficient reason" ([24], p. 302). In quantum physics, unlike classical physics, probability is not a consequence of our lack of knowledge, but rather it is objective probability that describes objective randomness. The wave function 'programs' the behavior of a quantum object, preventing it from making a transition to those $u_{n}$ for which $w_{n}=\left|c_{n}\right|^{2}=0$. In experimental terms, probability $\left|c_{n}\right|^{2}$ is the frequency of the event $\lambda_{n}$ (which is the eigenvalue of the operator $\hat{A}$ for the eigenfunction) observed over a large number of repetitions of the experiment. Now what is this repetition?

A particle, for example, an electron, is 'prepared' in a certain state $\Psi\left(x, y, z, t_{0}\right)$., and then its property $A$ is measured. Then we take the particle of the same kind in the same state and measure $A$ again. The experiment is then repeated a large (ideally, an infinite) number of times. As a result, according to von Mises's statistical definition of probability, the experimentalist will learn the frequencies with which the $\lambda_{1}, \lambda_{2}, \ldots$ are observed, applying the Born rule.

Thus, confirming predictions extracted from the wave function generally requires considering an ensemble of identically prepared particles.

III. Superposition principle. If $\Psi_{1}$ and $\Psi_{2}$ are the states of the system, then any linear combination thereof, $\Psi=c_{1} \Psi_{1}+c_{2} \Psi_{2}$, with $c_{1}, c_{2}$ being complex numbers, is also a possible state of the system.

IV. Corpuscular-wave dualism. The wave function of a quantum particle can be obtained by applying to a Fock vacuum state the operator of the local quantum field associated with the particle (the electron-positron field for the electron and the positron, the electromagnetic field for the photon, etc.). It is then clear why the wave function satisfies the Schrödinger equation and what the reason for and meaning of corpuscular-wave dualism are. The reason is that, even though we observe the particle as a pointlike object, the program for its evolution in time is determined by a quantized field that exists, in reality, as an element of the set of observables of a quantum object. Multiplying the absolute square of the wave function, which is the spatial probability distribution, by the electron charge yields (to within a normalization factor) the charge density of the electron field. Observing this density requires a state of an infinite number of electrons (which is an eigenstate for the frequency operator; see below), so that the wave properties of the electron are also observed as the properties of this ensemble of particles. In the truth, as shown by Everett [14, Graham [25], and Hartle [26], Born's rule allows a proof.

Indeed, consider an infinite set of states of a particle that have been identically prepared at 
different times (indexed 1, 2, . ) and also consider a wave function which is a von Neumann's infinite-dimensional tensor product:

$$
\left|\Psi^{\infty}\right\rangle=|\Psi, 1\rangle \otimes|\Psi, 2\rangle \otimes \ldots
$$

It can then be shown [25, 26] that the vector $\left|\Psi^{\infty}\right\rangle$ is an eigenfunction of a special operator $\hat{f}^{(k)}$, whose eigenvalue is the frequency of the outcome $\lambda_{k}$ obtained in measuring the property $A$ $\left(\hat{A} u_{n}=\lambda_{n} u_{n}\right)$, so that

$$
\hat{f}^{(k)}\left|\Psi^{\infty}\right\rangle=|\langle k \mid \Psi\rangle|^{2}\left|\Psi^{\infty}\right\rangle
$$

Here, we have resorted to the Dirac notation $|\langle k \mid \Psi\rangle|^{2}=\left|\left(u_{k}, \Psi\right)\right|^{2}$.

Given the above discussion of quantum physics postulates, what is the problem that splits the physics community? The answer is measurement. There are two key features to this concept.

A. Conversion of a pure state into a mixture of states. The question arises: Why is it that a quantum object, when not measured, evolves in such a way that its wave function changes deterministically in accordance with the Schrödinger equation — a process which is described by a unitary operator $U(t)$ - whereas in making a measurement the wave function collapses unpredictably into an eigenfunction of the observed quantity? Is it not true that the measurement, i.e., the interaction of the probe particle with the apparatus which, in turn, also consists of particles, is also described by the Schrödinger equation, i.e., by a unitary operator?

The following example will serve to illustrate why a measurement (or rather a first-kind measurement) violates unitary of the evolution.

Suppose there is a particle with a wave function $\Psi(x, y, z, t)$ and an instrument measuring the observable $A$, resided initially in the state $\chi_{0}\left(x_{a}, y_{a}, z_{a}, t\right)$. Upon making a measurement, considering the particle and the instrument to be quantum in nature, we should have

$$
\begin{aligned}
\Psi\left(x, y, z, x_{a}, y_{a}, z_{a}, t\right) & =\left(\sum_{n} c_{n} u_{n}(x, y, z, t)\right) \chi_{0}\left(x_{a}, y_{a}, z_{a}, t\right) \\
& \rightarrow \sum_{n} c_{n} u_{n}\left(x, y, z, t^{\prime}\right) \chi_{n}\left(x_{a}, y_{a}, z_{a}, t^{\prime}\right)
\end{aligned}
$$

where the functions $\chi_{n}$ correspond to various positions of the instrument 'pointer'.

Transition (3) requires a special kind of interaction, because we would generally have an expansion in terms of different basis functions:

$$
\sum_{n, k} c_{n k} u_{n} \chi_{k}
$$

which does not lead to a measurement. 
Thus, transition (3) proceeds unitary and occurs according to the Schrödinger equation.

The question to address next is: If the composite particle-instrument system is described at the time moment $t$ by the function (3), what is it that describes the particle as a subsystem? The answer is that, for orthogonal $u_{n}$, the subsystem has the density matrix

$$
\rho=\operatorname{diag}\left(\left|c_{1}\right|^{2},\left|c_{2}\right|^{2},\left|c_{3}\right|^{2}, \ldots\right)
$$

which is diagonal in the given basis. Now, what is the interpretation of this density matrix?

For an observer watching the subsystem, the density matrix $\rho$ describes a mixture of states, i.e., the observer will say that the particle is in a certain one of the set of states $u_{1}, u_{2}, \ldots, u_{N}$, the respective probabilities of the outcomes $u_{1}, u_{2}, \ldots$ being $\left|c_{1}\right|^{2},\left|c_{2}\right|^{2}, \ldots$ However, in this interpretation the system as a whole is no longer described by a pure state, superposition (3), but only by a mixture of states, the particle-instrument system being in states $u_{1}, \chi_{1}, u_{2}, \chi_{2}, \ldots$ with respective probabilities $\left|c_{1}\right|^{2},\left|c_{2}\right|^{2}, \ldots$

Thus, everything depends on whether or not the observer looks at the subsystem. If not - i.e., if only the system as a whole is observed - the states do not mix, and the system is, as before, described by a pure state.

Note that the very possibility of observation is important here. It is relative to the observer that a pure state turns into a mixed state, a situation which is, as de Broglie put it, the complimentarity of whole and part: knowing a part destroys the whole.

The conversion of a pure state described by a single wave function into a mixture of states with different wave functions constitutes a nonunitary operation contradictory to the Schrödinger equation. The net result of measurement is that the observer now knows ('registers') in which one of all the possible states the system ends up, i.e., of all the different $u_{n} \chi_{n}$ states only one - that which has been actually realized - is fixed. This last registration event is completely analogous to that in the classical physics, in which, after a measurement has been performed, the observer knows for certain that the instrument pointer has a definite position even though they do not know which one. But after the observer has looked at the instrument, only one of all the possibilities occurs. What makes things mysterious is the conversion of a pure state into a mixture, something which violates the ordinary unitary evolution of a quantum system.

In his book [27], V A Fock argues, quite rightly, that these two types of change undergone by the wave function reflect two types of change also occurring in classical physics: a change according to the equation of motion, and a change in the initial conditions.

The reduction of the wave packet leads, through the interference of the measuring instrument, 
to a change in the initial conditions for the subsequent evolution. Book [27], however, makes no mention of the fact that in classical physics this change in the initial conditions can be described in terms of the same classical physics. In quantum physics, however, this is not the case: the result of measurement cannot be obtained from the Schrödinger equation. The above problem was a subject of discussion.

The above problem was a subject of discussion between the present author and Vladimir Aleksandrovich Fock for two years after the publication of his book [27], two years which, alas, proved to be V A's last. There were plans to compile the Russian translations of major works on measurement problems in one book some day, with a preface on V A's own position on the subject. Unfortunately, these plans were cut short by his death.

B. Another problem concerning measurement is related to its special aspect in that it converts the numerically indefinite values of a physical quantity into a definite one. Unlike classical physics, this conversion is not simply the fixation of the value that existed prior to the observation and was only unknown to the observer, but rather the conversion of an 'objective indeterminacy' to a numerical value.

As we noted in Refs [28]-[30], the experimentally con- firmed violation of Bell's inequalities in quantum physics forbids speaking of the existence, prior to measurement, of the numerically definite values of properties described by noncommuting operators. Measurement does not fix the existence of a value that existed prior to it; it creates this value.

Another example to illustrate the nonexistence, prior to measurement, of the numerical values of observables (for a one- or more dimensional Hilbert space) is the Kochen-Specker example - a so-called quantum polyhedron [3].

We will now proceed by considering those interpretations of quantum physics which, in some way or another, accept its mathematical formalism. The pragmatic interpretation, which we already mentioned in the Introduction, is essentially that we should consider the quantum postulates as just the way things are and stop at this. But if we do not want to, there are two possibilities to consider, which are outlined in Sections 3 and 4.

\section{Copenhagen interpretation}

Originally proposed by Niels Bohr, Werner Heisenberg, and John von Neumann and further developed by Aage Bohr in the latter half of the 20th century [13], the Copenhagen interpretation can be summarized as the following set of principles. 
The world is divided into quantum objects and apparatuses, the former being described by quantum physics and the latter by classical physics [so that the results of measurements are described in terms of classical concepts and classical (Boolean) logic]. Notice that, while most of apparatuses themselves are macroobjects consisting of a large number of microparticles, this is not necessarily the case. In the Stern-Gerlach experiment, which measures the projection of the electron spin onto a certain direction, the role of the apparatus is played by silver ions which move in this direction or another along a classical trajectory, depending on the direction of the magnetic field and the value of the spin projection. While silver ions are not macroscopic bodies, under the conditions of the experiment their center of inertia moves along a classical trajectory and plays the role of a classical apparatus. The main property of the apparatus is that it enables the observer to measure only commuting observables, those which provide him with information about some property of the quantum object even though the apparatus consists of quantum objects. But of course, ultimately, even the quasiclassical ion trajectory is registered by a macroscopic apparatus - by a photographic film, for example.

The usual choice for such commuting apparatus observables is quasiclassical macro-observables, so that, due to decoherence phenomenon (which depends on the number of particles in the environment) the density matrix of the quantum object rapidly becomes diagonal. Those properties of quantum objects that are described by noncommuting operators take on their numerical values in additional experiments run with various instruments, but, if not via measurement, there are no fixed numbers to characterize the properties of a quantum object. Bohr's and Fock's concept of relativity with respect to the means of observation, which is about the 'emergence' of numerically definite properties upon making a measurement, is the most relevant to describe this situation. This concept first appeared in the special theory of relativity (STR) where, unlike Newtonian mechanics, the length of an object and the duration of a measurement process are not the attributes of the object itself but rather refer to the 'relation' of the observed object to another object associated with the observer - to an inertial reference frame. A reference frame, as a collection of rulers and clocks, is an 'apparatus' that measures length and duration of the process as the 'relation' to this apparatus. These 'relations' change as the reference frame changes, which manifests itself in the Lorentz contraction of the length scale and time. There is, of course, a 'proper' reference frame, which reflects the 'self-relation'; nonetheless, already in STR one cannot speak of a definite length and a definite duration without identifying the reference frame.

In quantum physics, a quantum object is 'in and of itself' described by operators rather than by numbers (as in classical physics), so that it 'objectively' represents a set of observable operators. 
As mentioned in Section 2, these operators include generators of the Poincaré group, those of local quantized fields and various gauge transformation charges.

With respect to an apparatus described classically - that is, in the language of numbers — operators 'turn into' numbers, i.e., into the eigenvalues of the operators. The wave function characterizes a certain special 'relation' between an apparatus and the quantum object and, in this sense, describes both of them together. The wave function is defined in combination with the 'preparing' or 'measuring' apparatus. Reference [31] is another work to highlight the importance in modern physics of such a category as 'relation'. The situation that arose in quantum physics is occasionally described by some authors [32] in terms of there being 'objective indeterminacy', 'objective randomness', and 'objective probability' in the microworld. To reemphasize, a quantum object 'in itself' is not generally characterized by any number. For example, the presence of a coordinate operator instead of a number indicating the position of the object can be interpreted as 'objective indeterminacy' of the position of the quantum object in space. It is only through measurement that such a number-defined position emerges. Unlike 'subjective indeterminacy', the term 'objective indeterminacy' implies that indeterminacy is not related to our lack of knowledge (as is the case in classical physics) but rather is a property of the object itself. Heisenberg's words [33], speaking of quantum reality as "something in itself" brings quantum physics closer to Platonism.

Description on the language of operators implies, in a sense, the existence of 'a coordinate in general', of 'a momentum in general', of 'a particle in general' (the principle of identity of particles), etc. This is, in fact, medieval realism, known for its opposition to nominalism on the question of the existence of general notions (say, a man in general or a woman in general - not only specific Bobs or Alices). As is known, it was nominalism which got the upper hand.

But in quantum mechanics, the principle of identity of particles states that the members of a system of electrons cannot, due to their being identical, be assigned an individual 'name' such as first, second, etc., and the only thing we can speak of is the number of electrons. All this calls for more attention to realism and its ensuing Platonism.

Finally, there is, according to Finkelstein [34, yet another language to speak. A quantum object is a set of plurality of 'acts' that are described by operators, but the apparatus turns 'acts' into 'facts' that are characterized by commuting operators and are therefore described by numbers.

The time evolution of a quantum object is described in a natural way in the Heisenberg representation. Thus, in the quantum theory of fields, when describing the double commutator of the Hamiltonian with the field, the time evolution of the field leads in a natural way to quantized 
field equations: the Klein-Fock equation for a scalar field, the Dirac equation for a spinor field, etc. However, in the Schrödinger representation, which is usually equivalent to the Heisenberg representation, one should speak of the apparatus, without which the wave function is not defined. From the quantum field theoretical perspective, the wave function results from the application of the field operator to a vacuum, and if the apparatus moves noninertially — for example, with a constant acceleration - the Fulling-Unruh effect arises [35] and equivalence is violated. A similar nonequivalence also arises when as the Fock vacuum for the operators of a field with a certain mass is taken the vacuum of a field with a different mass [36]. This seems to make the Heisenberg representation preferable for describing the evolution of a quantum system.

Now let us look in more detail at the problem of wave packet reduction.

A number of attempts have been made to interpret this concept. As is known, von Neumann [24] linked the reduction phenomenon to the consciousness of the observer in the sense that any apparatus extends the sense organs of the observer who receives information about the world around them. Any apparatus can be considered to consist of quantum objects - atoms and molecules - and, hence, can be treated by the laws of quantum physics - assuming that the 'principle of transferring the boundary' is valid.

The object-apparatus boundary can be brought toward the observer's eyes, which are considered classical, and at the same time, what was previously considered to be an apparatus is now combined with the object being studied into a common quantum system. According to von Neumann, the result of the observation is the same wherever the boundary is drawn. Although the boundary can be moved further into the brain of the observer, consciousness or the 'abstract I' will always be present as a subject of cognition receiving information.

The apparatus-object boundary may not necessarily be drawn in the present. Because the apparatus is treated classically, it is possible, by applying the laws of classical physics with its determinism, to argue that the apparatus at the time of measurement had produced - even before the observer looked at it - a certain definite result, though unknown to the observer. The logic here is that the observers would see a quite definite outcome if they looked in the past (not now!) at the object which they call the apparatus and which interacted via a special kind of interaction (the 'measurement Hamiltonian') with the microobject.

Von Neumann's view on the role of the subject of cognition in the process of wave packet reduction has been criticized for 'solipsism'. Reference [37] argues, not without irony, that, according to von Neumann's interpretation, "every one of us should be of the view that he alone is the observer of significance, whereas the rest of the Universe and its residents satisfy the Schrödinger equation 
at any time - except when he does the measurement."

There is a well-known philosophical argument [38] to advance against this, though, according to which the notion of a number does not apply to the subject of cognition. The only subject of knowledge is I. All the rest is objects for the I. It goes without saying that this I should not be identified with me as Alice, Bob or some other objects both for myself and for others. Megalomania occurs only when I, Alice, am the Subject - unlike Bob who is no more than an object. Every cognizing observer is I as the subject of cognition. Because I is the one and only, it follows that what I see as a result of quantum observation will be the same for other observers. This is why there is no place for the Wigner's-friend type paradox, according to which, if the reduction event is due to the consciousness of the observer, then different people — say, Wigner and his friend — could see different results. This paradox assumes a multitude of consciousnesses, which is inconsistent with von Neumann's reasoning. Given his awareness of and connection with the great German philosophical tradition, it seems von Neumann knew well what he meant when using the term 'abstract I of the observer'.

It can be said without any philosophical argumentation, though, that, according to the rules of quantum mechanics, for the first-kind observations - in which the object after the measurement resides in an eigenstate of the observable operator - there is no inconsistency between the measurements of two observers if the measurements are conducted at different instants of time. Whether the measurement was first done by Wigner and then by his friend, or the other way round, the result would be the same for both.

This view on the role of consciousness is very much shared by Schrödinger. To quote from his What is Life? [39]: "'I', when taken in the broadest sense of the word - i.e., every conscientious mind that sometimes said or felt ' $\mathrm{I}$ ' — is nothing other than a subject capable of controling the 'motion of atoms' according to the laws of nature."

Later on, F London and E Bauer [40], E Wigner [41], and R Penrose [42] furthered the idea that it is the observer's consciousness that causes the reduction of the wave packet.

Here is the reasoning of London and Bauer. Suppose a complex system - quantum object X, apparatus $\mathrm{Y}$, and observer $\mathrm{Z}$ - is described, following the interaction of the quantum object with the apparatus, by the wave function

$$
|\Psi(x, y, z)\rangle=\sum_{k} a_{k}\left|u_{k}(x)\right\rangle \otimes\left|v_{k}(y)\right\rangle \otimes\left|w_{k}(z)\right\rangle,
$$

where $\left|w_{k}(z)\right\rangle$ are various states of the observer. It should be noted that, unlike von Neumann's observer, the observer $\mathrm{Z}$ is objectivized and described by a wave function. (Clearly, for philosophers 
like Schopenhauer and N A Berdyaev, the immediate reaction would be that what we are speaking of is not the subject of cognition but consciousness as an inalienable property of the observer.)

The observer as a subsystem of the complex system is described by the density matrix which, however, is not as yet interpreted as a mixture of states. But here is what the authors of Ref. [40] go on to say: "For him (the observer), only the object $\mathrm{X}$ and the apparatus $\mathrm{Y}$ are things from the outside world. To himself, on the contrary, he is in a special kind of relation: he has a well-known ability, which can be called the ability of self-observation (introspection), and so needs no mediator to identify the state he is in. This self-cognition enables him to break the chain of statistical links which are expressed by the wave function $\sum_{k} a_{k}\left|u_{k}(x)\right\rangle \otimes\left|v_{k}(y)\right\rangle \otimes\left|w_{k}(z)\right\rangle$ and to say: I am in the state $\left|w_{k}(z)\right\rangle$, or I see $g=g_{k}$ ( $g$ being the reading of the apparatus) or directly $f=f_{k}$ ( $f$ being a property of the quantum object)".

Thus, it is the observer's consciousness which is responsible for the transformation of the density matrix into a mixture of pure states, so that introspection produces a new wave function for the object, namely $\left|u_{k}(x)\right\rangle$. Consciousness, unlike unconsciousness, can manifest itself in a certain pure state from the set $w_{k}(z)$, but not in a state described by the density matrix.

It is relevant here to discuss the Wigner's friend paradox. A transition into a pure state is not due to an objective, outside-of-the-observer process, but due to something like the observer's choice. But then, why cannot another observer make a different choice? It is important to note, however, that much depends on who is the first and who is the second when do taking a measurement. If the reduction to a certain wave function was performed by the first observer, then, according to the properties of the measurement Hamiltonian, the second observer will obtain the same wave function as the first when taking the same measurement. (Everett [14] followed the same reasoning when arguing why different observers find themselves in one and the same of all possible Everett's worlds.) Squires [43] and Menskii [21, 44, 45] closely follow this view on the role of consciousness.

Clearly, a proof of the validity of this interpretation should be looked for in psychology and physiology, disciplines for which the main problem yet to be solved is the psychophysical one of how consciousness (that is, something nonmaterial and described in psychological terms) controls the body which is described in physical terms. Whether quantum theory can help in solving this problem remains an open question.

An objection to this view may be seen in the question $\mathrm{R}$ Penrose [46] asked regarding the Everett interpretation: "How can we know that consciousness cannot be conscious of itself as something described by the density matrix or by a number of wave functions?"

To add to the above, the indeterminism and probability that arise during measurement are 
due to the observer; they do not exist in the objective quantum world. But if this probability is objective, then it reflects the uncontrollable randomness in how the observer makes their choice. The observer cannot control this randomness and, even though the randomness has no relation to their lack of knowledge, as in the classical physics, it is their choice that determines it.

In this connection, the question posed by M B Menskii [21] is worth considering: Is it possible that the observer, with his subconscious access to all the possibilities offered by superposition, can make a choice which benefits him?

After repeating this many times, he will end up violating the probabilistic predictions of quantum physics. While this choice is possible in single individual cases, in the multiple repetition scenario the action of consciousness cannot be distinguished from a pure coincidence.

Finally, if the observer is still unable to make such a choice, it seems natural to conjecture that this choice will be made by the microparticle - but only with respect to the observer who receives information about this particle. This is reminiscent of the free will of the electron Dirac spoke of in the early days of elaborating quantum physics.

The role of reception of information by the observer is particularly manifested in what is known as 'negative' experiments. The first such example was given by Renninger [47].

Suppose a source of charged particles (electrons) is placed inside a sphere with a hole in it, coated with a scintillating material, and surrounded by a larger sphere. An electron striking the smaller sphere causes a light flash (scintillation), and information about the occurrence (or otherwise) of the flash on this sphere is transferred to the observer (by means of electromagnetic radiation). Suppose, however, that although an electron has flown out of the source, no scintillation was observed. The observer will then say that the electron has escaped through the hole. Due to the Heisenberg uncertainty relation, the momentum of the escaping electron is different from that in the case of no negative observation and also from the momentum the electron had when leaving the source. Thus, the reduction of the wave packet occurred even though the electron was not observed in a certain region of space. A similar macroscopic situation would arise if the observer knew that a train was going to leave Chicago with a known speed, maybe heading for Los Angeles, maybe heading for New York, and a failure to observe the train passing an intermediate station would change the speed of a train going from New York.

Unlike the defenders of the reduction model discussed above, Roger Penrose advocates the idea of 'objective reduction' as a certain physical process violating the unitarity of evolution [42, 46]. Given the notion of applying relativity principle to the means of measurement, this is reminiscent of the Lorentz-Fitzgerald idea that the Lorentz contraction due to changing a reference frame can 
be attributed to the contracting effect of electromagnetic forces rather than being interpreted as a geometric property of the Minkowski four-dimensional spacetime.

Penrose suggests a process in quantum gravity to illustrate his point. He does not believe that different spacetimes can form a superposition and argues, therefore, that quantum gravitation violates unitarity.

Quantum gravitation should perhaps be taken into account when considering interaction with macroscopic bodies. That macroscopic bodies may require the quantization of gravitation for their description is a long-discussed idea. To illustrate, if we write out the de Broglie wavelength of a truck (or rather of its center of mass), we find that the period of such vibrations is much less than Planck's time, suggesting the necessity of the quantization of gravitation.

Unfortunately, quantum gravitation has not yet been developed, so Penrose's idea remains just a hypothesis.

Some support for the idea of considering consciousness was drawn from a field which came to be known as 'quantum logic' (a subfield of quantum axiomatics).

J von Neumann, the cofounder of quantum mechanics, came across the question of why the properties of quantum objects are described by Hilbert space operators [24], Ch. 6. In his joint work [48] with Birkhoff, it has been found that this is due to the existence of a special mathematical structure, a complete orthomodular lattice (for an exact definition, see, for example, book [28]), which possesses the properties of a logical structure with operations 'AND' (conjunction, $\wedge$ ), 'OR' (disjunction, $\vee$ ), and 'NOT' (negation, $\neq$ ), for which the properties 'AND', 'OR' does not hold. In Aristotelian (or Boolean) logic, we always have

$$
A \wedge(B \vee C)=(A \wedge B) \vee(A \wedge C)
$$

However, if

$$
A \wedge(B \vee C) \neq(A \wedge B) \vee(A \wedge C)
$$

this leads to quantum theory with its noncommuting operators. The original analysis in paper [48 treated the simple case of finite-dimensional Hilbert space. Subsequent work by Jauch [49], Piron [50], and others from the Swiss theoretical school extended the analysis to include an infinitedimensional Hilbert space.

Whereas the logic of our consciousness is distributive (or Boolean), that operating in the world is of a different — nonhuman, non-Boolean — nature. Logic, like geometry with its many nonEuclidean versions, is not unique. Other logics, like logics of the behavior of some objects, can be revealed experimentally, for which purpose it is necessary to design experiments in which the 
properties of the objects could be measured in such a way that, at the same time, more complex properties, considered as conjunction and disjunction of the original properties, can also be established. Thereafter, it is also necessary to check whether the distributivity property holds for conjunction and disjunction.

Non-Boolean logic allows one to argue that a pointlike electron with a definite momentum (property A) flying through a screen with two slits (properties B, C), i.e., $A \wedge(B \vee C$ ) is not the same as when an electron (while remaining pointlike) flies either through slit C or through slit B.

The following two aspects reveal the role of consciousness in 'quantum logic':

(a) Boolean consciousness, receiving information about the microworld, projects the nonBoolean quantum-logical world onto a Boolean structure. The nonisomorphic nature of these structures manifests itself in that at different instants of time consciousness chooses some Boolean substructures corresponding to the sets of the commuting operators of the quantum object, so that the noncommuting operators are measured at different instants of time in order to obtain information about the quantum object. Note that time plays a very important role here - as if the observer would invented time expressly to identify the quantum object [51];

(b) Boolean consciousness defines the function of truth (difference between true and false events) on the observables of a Boolean subsystem. However, unlike the truth function in classical physics, prior to observation this function is not generally defined on a nondistributive lattice (KochenSpecker theorem [2]) and occurs randomly, which is the reason for quantum indeterminacy and for relativity with respect to observation.

Now the question can be asked: If non-Boolean logic is nonhuman, how can we think and speculate about it at all?

Here we face a situation quite similar to that with extra dimensions. Although the fourth and fifth dimensions are beyond our imagination, analytical geometry makes it possible to translate the question of multidimensional images into that of algebraic equations that represent these images without the need for geometrical imagination. It is appropriate here to recall L D Landau's words that humans can comprehend things which they cannot imagine. By the same token, non-Boolean logic is studied by replacing logical operations by lattice operations isomorphic to — but not identical to - them.

A simple example of a particle with spin $S=1 / 2$, characterized by two spin projections onto the $x$-, $y$-axes [28], will illustrate the above point without using complex mathematical reasoning (which, in turn, assume knowledge of the relevant definitions). Let us draw the Hasse diagram for a quantum-logical lattice (Fig. 1). From this diagram it is seen that 


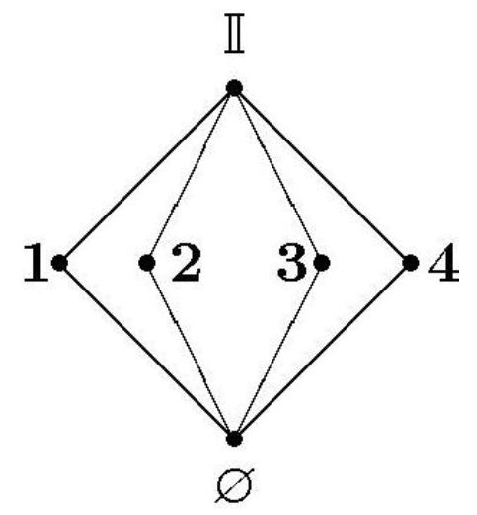

Figure 1: Hasse diagram. Slashed circle $\varnothing$ denotes 'always false', and I denotes 'always true'. Dots 1, 2, 3, 4, called logic atoms, denote: $1-S_{x}=1 / 2,2-S_{x}=-1 / 2,3-S_{y}=1 / 2$, and $4-$ $S_{y}=-1 / 2$. Lines converging at the bottom and at the top denote conjunction and disjunction, respectively. Upward line stands for 'it follows'.

$$
\begin{aligned}
& 1 \wedge 2=2 \wedge 3=3 \wedge 4=2 \wedge 4=\varnothing, \\
& 1 \vee 2=2 \vee 3=3 \vee 4=1 \vee 4=I .
\end{aligned}
$$

The distributivity property, however, does not hold:

$$
1 \wedge(2 \vee 3)=1 \wedge I=1 \neq(1 \wedge 2) \vee(1 \wedge 3)=\varnothing \vee \varnothing=\varnothing
$$

(It is assumed, in accordance with the properties of the lattice, that $1 \wedge I=1$.) As a possible truth function for this simple lattice, the observer could introduce, for example, the following function: 1 is true and 2, 3, 4 are false as incompatible with 1 . Notice, incidentally, that for more complex quantum systems, in particular, for those with spin 1, it may happen that, unlike a Boolean lattice, such a function cannot be introduced at all (as Kochen-Specker and Peres examples illustrate [3]). The simple example we have given shows, however, that the falsity of 3 and 4 implies that 3 or 4 are always true, which is, of course, at odds with our logic.

Therefore, a 'Boolean observer' sees a Boolean sublattice of elements, which in no way contradicts her/his logic. At the next instant of time, she singles out another Boolean system, 3 and 4, and assigns a new truth function to it: 3 is true, 4 is false or 4 is true, 3 is false. All this cannot be otherwise, given her Boolean consciousness. She cannot, after all, allow a situation with 3, 4 both false and 3 or 4 true. But, then, the 'Booleanization' of non-Boolean logic leads to an indeterminate definition of truth and to relativity with respect to the means of measurement.

Thus, it is untenable to argue that truth and falsity exist in a quantum system prior to measurement and that all the difference between a classical and a quantum object is nondistributivity with 
observer-independent truth values. One cannot speak of any definite measurement-independent values of physical quantities described by noncommuting operators.

Nondistributivity, as is easily seen and as was realized already by Birkhoff and von Neumann, is inconsistent with Kolmogorov's probability axiomatics and requires introducing the probability amplitude as a new tool for describing randomness for non-Boolean lattices. It is beyond the scope of this paper, though, to discuss this in more detail.

The brief discussion above concerned the quantum- logical version of the Copenhagen interpretation, a version which enables one to say, in addition to what London and Bauer, and von Neumann said, that the important function of consciousness - and one without which it would hardly deserve its name - is to distinguish between true and false. But, true and false in Boolean classical physics are distinguished 'objectively' without any mention of consciousness. In quantum physics, on the contrary, true and false emerge in microobjects when their properties become realizable and do not exist otherwise. However, what consciousness conceives as true and false is determined not by consciousness itself but by an object external to it, and is determined in a random way, with a probability calculated on a Boolean substructure with the aid of the wave function.

There is, finally, a view, often associated with some statements by Niels Bohr, that there is something in macroscopic bodies which prevents applying quantum theory to them to its full extent, so that consciousness plays no special role and the readings of macroscopic instruments are the same as they would be in the absence of it.

At one time there was a belief that macroscopic bodies differ from microscopic objects in that the former consist of a large number of microscopic objects and that perhaps we have a situation analogous to that of passage from statistical physics to thermodynamics. Then classical physics, like thermodynamics, should be more accurate as the number of particles in the macroscopic object increases. This hope, however, was dashed by Hepp's theorem [52] asserting that, for any quantum system of many (but a finite number of) particles, there is always an observable whose operator does not commute with the rest of the operators, and whose measurement will lead to an arbitrarily large departure from classical theory. Only in the case of an actually infinite number of particles is it possible to obtain a classical theory in which interference effects are absent due to the emergence of superselection rules. Real macroscopic objects always consist of a finite number of microparticles.

Another attempt to turn from considering microobjects to considering macroobjects is based on the idea of decoherence. The crux of the idea is essentially that a macrobody not only consists of a large number of particles but it also interacts with a large number of particles in its environment. 
If we take an average over the ensemble of particles in the environment, and if, in doing so, we focus our attention only on describing the macrobody, then a certain system of mutually commuting quasiclassical observables can be singled out so that the evolution of such a system can be described on this basis to good accuracy in terms of classical physics. So, if we are interested to know the position of the pointer of the macroapparatus, a distinction should be made between the external environment and the internal one containing a macroscopic number of particles.

Quantum-mechanically, the position of the pointer's center of gravity is influenced both by the interaction with the particles of the pointer itself and by the interaction with particles outside it. The pointer as a system is described by a matrix density which is within a short time diagonalized in a quasiclassical basis. Interference terms, whose presence is what distinguishes a quantum system from a classical one, rapidly vanish — within a time scale which, for certain cases (e.g., for a system of oscillators), is determined by an exponential with the number of environmental particles in its exponent. This is exactly the reason why we do not watch the interference between the alive state and the dead state of Schrödinger's famous cat. (For a more detailed discussion of decoherence, see Ref. [53].)

Now, does decoherence solve the measurement problem? Many believe that it does not. J S Bell, for one, asks what there is in nature to make a quantum system with a macrobody say: "I am a system, and you are my environment, and we will average over you" [54]. It has also been noted that the diagonalization of the density matrix without the observation of the subsystem does not yet imply the emergence of a mixture of states for the whole system, the point we have discussed above in connection with the violation of unitarity in measurement. All decoherence does is to explain why we human-observers do not see macrobodies interfere.

Wojciech Zurek [55] and M B Menskii [21], in connection with the existence of a selected quasiclassical basis of the eigenfunctions of commuting macroobservables, make an attempt to relate this choice to evolutionary biological selection. In this approach, not only do living beings adapt themselves to nature, but nature also adapts itself to the living beings that observe it. How life is viewed from the perspective of physics was also discussed in Refs [56, 57]. Living beings turn out to be more adapted when they do not ask 'dangerous' questions to the macroworld in their neighborhood, thereby choosing a special 'reference frame in a Hilbert space', so that, due to decoherence, classical physics with its determinism and predictability turns out to be approximately valid.

Other bases also exist, in which Schrödinger's cats in the state of interference walk by themselves next to us. So we see that quantum physics with all its unpredictability invades the macroworld. 
The present author discussed a similar idea in connection with certain problems in psychology [58]. Decoherence also plays an important role in how we conceive the macroscopic Universe which, while primordially quantized, seems to us to be classical with respect to a quasiclassical basis.

It is important to note that, for macrobodies regarded as quantum objects consisting of a large number of particles interacting with a large number of particles in the environment, decoherence is a consequence of the 'entanglement' between the states of particles in the macrobody and those in its environment, so that the body itself is described by a density matrix. This means that a macroapparatus, like any 'classical' body, from the standpoint of quantum physics is not at all isolated from the surrounding world.

Furthermore, from a microscopic point of view, an apparatus macroscopically isolated from the Universe is not at all isolated from it but, indeed, due to the entanglement of states, is tied to it more strongly than an individual quantum particle. This new understanding of classical macroobjects from the quantum perspective is an important recent development.

\section{Everett interpretation}

Much popularity has recently been gained by the Everett interpretation [14]. The idea was disseminated in 1957 and found initially support from John Wheeler in his paper [15] published in the same journal issue.

Hugh Everett - a Princeton graduate, theoretical physicist, and great mathematical talent defended his Ph.D. thesis on a new interpretation of quantum mechanics based on the assumed objectivity of the wave function. The thesis was published in 1973 [16], much later than his famous paper. After unsuccessful (in his view) discussions with prominent physicists, he left physics to join a secret Pentagon department, where his job was the mathematical modeling of nuclear war strategies; this included, in particular, assessing the possible consequences of radioactive contamination and the optimization of nuclear attacks on the USSR. He was unsociable and had a reputation, perhaps exaggerated [59], for drinking. He died at the age of 51 in 1982. Of his two children, his son Mark became a rock star, and his daughter Elizabeth committed suicide by overdosing on sleeping pills in 1996. Her suicide note read that she was "going to join her father in another universe" 60].

The Everett interpretation came to be known as the 'many-worlds' or 'many-universes' interpretation (which, incidentally, explains poor Elisabeth's note).

A few background words first. David Deutsch, one of the most dedicated Everettians, claimed that the idea of many worlds described by a single wave function was first mentioned by Schrödinger 
in one of his lectures, with the remark that "the audience will probably consider me crazy"; anyway, he published nothing on the subject [61].

After his 1957 publication, Everett went to Copenhagen to discuss his views with Bohr. There, however, his theory received a rather chilly response. The most incisive was Rosenfeld, who wrote later to Bell that the assumption of a universal wave function for the Universe would mean that "our view of the Universe is that of God" 62].

Nor were such founders of quantum physics as Dirac, Wigner, and Feynman anything more than lukewarm. Feynman, in particular, wrote [62]: "The concept of a universal wave function has serious conceptual difficulties. This is so since this function must contain amplitudes for all possible worlds depending on all quantum-mechanical possibilities in the past and, thus, one is forced to believe in the equal reality [sic!] of an infinity of possible worlds."

Norbert Wiener doubted the possibility of introducing the Lebegue measure in a Hilbert space and advised Everett to publish his paper as a "comment on current debates on quantum theory rather than a definite result" 62.

The situation changed, however, when Bryce DeWitt derived what came to be known as the Wheeler-DeWitt equation for the wave function of the Universe. From that time on, DeWitt was an active defendant and disseminator of the Everett interpretation. It should be noted that Wheeler also originally supported the Everett interpretation, in the hope that it would be of use in developing quantum gravitation. However, Wheeler denounced this interpretation in 1977 as "introducing an infinite number of unobservables as metaphysical baggage" and converted to the von Neumann-Wigner interpretation, in which the consciousness of the observer is not described by a wave function 62 .

Bell's criticism was that the Everett interpretation "has many pasts and many futures" [54].

All this notwithstanding, DeWitt's work triggered activity in 'quantum cosmology', in which it is postulated at the outset that there is a certain Universe wave function which exists whether or not any observer is present and which has the same objective status as an electromagnetic field.

Such cosmologists as Stephen Hawking and Alexander Vilenkin use this concept in their science and popular science publications to avoid the problem of the primordial singularity of the Universe. While Vilenkin is quite unequivocal about his preferred interpretation (which is Everett's; see Ref. [19]), Hawking is somewhat vague and postulates his Universe wave function as an integral for a certain real (but non-Feynman) measure over various Euclidean universes.

It is clear, however, that discarding the Everett interpretation as false also casts strong doubts on quantum cosmology studies, unless, of course, we agree with Wheeler that consciousness is not 
part of the Universe, and that all the Universe is defined with respect to this consciousness according to his concept of the 'participating Universe'. But then, as with any version of the Copenhagen interpretation, the preparation and measurement of the wave functions and the observer-induced wave packet collapse — that is, the concepts ignored by the quantum cosmologists — should be invoked.

Advocates of the Everett interpretation include D Deutsch (UK) [63], B Carter (Australia) 64], M Tegmark (US) [65, Don Page (Canada) 66], M B Menskii (Russia) [20, 21, and Lev Vaidman (Israel) 67.

In 2007, a number of events commemorated the 50th anniversary of Everett's seminal paper, including a special Nature issue [68, a popular article in the Scientific American [69], two international conferences, and a BBC film on parallel universes. Note also that the Everett parallel universes are being promoted in science literature by supporters of string theory, 'the theory of EVERYTHING' (see, for example, Ref. [70]).

We will now proceed to directly discuss the Everett interpretation. In doing so, we will quote and refer to both Everett himself and the current Everettians. From its very start, the Everett interpretation positioned itself as opposing the Copenhagen interpretation.

(1) A wave function possesses the same objective reality - and is an observer-independent as an electromagnetic field.

(2) There is a universal wave function, i.e., the wave function of the Universe. In addition, Tegmark, an Everettian, says that individual particles have 'epistemological' wave functions, which are what experimentalists deal with; however, these functions also exist objectively [65].

(3) The wave function satisfies the Schrödinger equation and evolves unitarily. There is no need to postulate any wave packet reduction - i.e., a nonunitary operation - because all the observed properties follow from the Schrödinger equation.

(4) A measurement of a physical quantity in the case of the superposition of wave functions 'splits' the initial states of both the observer and the particle into a multitude of states belonging to different and noninteracting 'worlds' or universes. In addition to the splitting, there is a possibility that, due to the time reversal of the Schrödinger equation, the 'worlds' will merge, which distinguishes this theory from standard quantum physics, in which measurement violates time reversal. Therefore, the statement that the Everett interpretation leads to the same consequences as the Copenhagen interpretation is, of course, wrong. The only question is how to check this experimentally.

(5) Quantum mechanics applies to both microscopic and macroscopic phenomena. Classical 
physics provides an approximate description of those quantum objects with a large number of particles that satisfy the decoherence property.

Actually, it is clear why Schrödinger could give his full support to the above points. There indeed are arguments which Schrödinger could make against those criticizing his view that waves exist objectively and the electron constitutes a wave. His critics argued that the electron is always observed as a pointlike particle and that it is different from the electromagnetic wave, for which different radio receivers detect something at any point of its front. An electron is not observed as smeared over any front surface and, hence, the electron wave is a 'probability wave' for finding the electron at any place in space.

Schrödinger could object, following Everett, that an electron, while remaining pointlike particle, resides simultaneously (and, of course, without any self-multiplication) in different places of space in different worlds - the worlds in which the states of the observer are also different and correspond to different observational results, and which are independent for different states of the observer and not interacting between themselves.

A few words on Everett's paper [14] are in order. All the mathematical formulas it contains are known in ordinary quantum mechanics as well. Where the difference lies is in how they are interpreted. Of key importance is the concept of the 'relative wave function' of a subsystem of a quantum system. If a complex system $\mathrm{S}$ consists of two subsystems, $\mathrm{S}_{1}$ and $\mathrm{S}_{2}$, the Hilbert space for $\mathrm{S}$ is the tensor product of the Hilbert spaces of the subsystems. If these spaces contain complete orthonormalized sets $\left\{\xi_{i}^{\mathrm{S}_{1}}\right\},\left\{\eta_{j}^{\mathrm{S}_{2}}\right\}$, the total state of the system $\mathrm{S}$ can be represented as

$$
\Psi^{\mathrm{S}}=\sum_{i, j} a_{i j} \xi_{i}^{\mathrm{S}_{1}} \eta_{j}^{\mathrm{S}_{2}}
$$

From this it follows that, although $\mathrm{S}$ occupies a definite state $\Psi_{\mathrm{S}}$, the subsystems are not in definite states with respect to each other. However, for a certain choice of state for one subsystem, we can determine the corresponding relative state of the other. For example, choosing $\xi_{k}$ as a state of $\mathrm{S}_{1}$, the relative state in $\mathrm{S}_{2}$ has the form

$$
\Psi\left(\mathrm{S}_{2} ; \operatorname{rel}\left(\xi_{k}, \mathrm{~S}_{1}\right)\right)=N_{k} \sum_{j} a_{k j} \eta_{j}^{\mathrm{S}_{2}}
$$

with $N_{k}$ being the normalization factor. Paper [14], therefore, goes on to argue: "It is meaningless to talk about the absolute state of a subsystem - one can only talk about the state relative to a given state of the another subsystem."

Comparing this description with the situation in the theory of relativity, where the length of a body is defined relative to a reference frame and is otherwise indefinite, we note — by considering the 
system particle-apparatus-observer, in which, according to Everett, the observer is also described by a wave function - that the observer's wave function is defined relative to the apparatus wave function, which, in turn, is defined relative to the wave function of the particle. It should be noted, though, that prior to measurement, the observer - according to equation (10) of Everett's paper — was characterized by a certain 'absolute' wave function determined by the memory of events, so that the system involving the observer plus the quantum object before their interaction during the measurement is, according to the measurement Hamiltonian, described by (Eqn (10) in Ref. [14])

$$
\Psi^{\mathrm{S}+\mathrm{O}}=\Phi_{i} \Psi^{\mathrm{O}}[\ldots]
$$

We emphasize the following properties concerning the measurement of a quantity described by the operator of the observable $\hat{A}$ with eigenfunctions $\Phi_{i}$.

If the quantum system was found itself in an eigenstate of the operator $\hat{A}$, then, after the measurement, the total wave function transforms into the function (Eqn (11) in Ref. [14])

$$
\Psi^{\mathrm{S}+\mathrm{O}}=\Phi_{i} \Psi^{\mathrm{O}}\left[\ldots, \alpha_{i}\right]
$$

However, if the system resides initially in a superposition of eigenstates, $\sum_{i} a_{i} \Phi_{i}$, the final state after the measurement is given by (Eqn (12) in Ref. [14])

$$
\Psi^{\mathrm{S}+\mathrm{O}}=\sum_{i} a_{i} \Phi_{i} \Psi^{\mathrm{O}}\left[\ldots, \alpha_{i}\right]
$$

where the result $\alpha_{i}$ appears in the memory of the observer as an apparatus reading and is associated with that eigenvalue of the operator $\hat{A}$ which corresponds to the eigenfunction $\Phi_{i}$ of this operator.

And here we come to the important point in interpreting this obvious property of measurement. Everett insists on the following.

(1) Quantum physics describes all happening in microworld and macroworld alike. There is no need to follow Bohr in dividing the world into classical and quantum domains and describing the macroapparatus and the observer classically and the microobject quantum mechanically.

(2) Consciousness is totally irrelevant; the observer can be replaced by an automation or by a computer with memory.

(3) All the terms in superposition (12) are equally real. The wave function collapse concept is altogether unnecessary. In particular, it cannot be argued, following Copenhagen style, that, of all the sum, only one term is realized with a probability determined by the Born formula as a result of the measurement. The initial wave function undergoes a 'splitting' into superposition 
of states (12), with each term describing a real 'world' (or branch) in which the observer views a definite, world-specific measurement result.

(4) The worlds do not interact with each other, so an observer in one world knows nothing of another world.

It is shown further that, when measuring the same quantity again, the observer in a fixed world will, due to the unitary evolution in each branch, survey the same result.

Next, the concept of a measure for the superposition $\sum_{i} a_{i} \Phi_{i}=\alpha \Phi^{\prime}$, namely

$$
m(\alpha)=\sum_{i=1}^{n} m\left(\alpha_{i}\right)
$$

is introduced and it is proved that the countable additivity of this measure fixes it as

$$
m\left(\alpha_{i}\right)=c a_{i}^{*} a_{i}
$$

where $c$ is a constant - that is, we arrive at the Born probability formula of quantum physics.

Finally, an analysis is made of a system of many quantum objects, $\mathrm{S}_{1}, \mathrm{~S}_{2}, \ldots, \mathrm{S}_{\mathrm{n}}$, identical in the sense of being all described by the same wave function $\Psi^{\mathrm{S}_{1}}=\Psi^{\mathrm{S}_{2}}=\ldots \Psi^{\mathrm{S}_{\mathrm{n}}}=\sum_{i} a_{i} \Phi_{i}$. Before measurement, the wave function of the system and the observer with memory is written out as

$$
\Psi^{\mathrm{S}_{1}+\mathrm{S}_{2}+\ldots+\mathrm{S}_{\mathrm{n}}+\mathrm{O}}=\Psi^{\mathrm{S}_{1}} \Psi^{\mathrm{S}_{2}} \ldots \Psi^{\mathrm{S}_{n}} \Psi^{\mathrm{O}}[\ldots]
$$

After $r(r \leq n)$ measurements, assuming that the measurements of one and the same quantity $A$ are performed in the order $\mathrm{S}_{1}, \mathrm{~S}_{2}, \ldots, \mathrm{S}_{r}$, we obtain for $\mathrm{n}$ systems the following expression

$$
\Psi_{r}=\sum_{i, j, \ldots, k} a_{i} a_{j} \ldots a_{k} \Phi_{i}^{\mathrm{S}_{1}} \Phi_{j}^{\mathrm{S}_{2}} \ldots \Phi_{k}^{\mathrm{S}_{r}} \Psi^{\mathrm{S}_{r+1}} \ldots \Psi^{\mathrm{S}_{n}} \Psi^{\mathrm{O}}\left[\alpha_{i}^{1} \alpha_{j}^{2} \ldots \alpha_{k}^{r}\right]
$$

The function $\Psi_{r}$ is interpreted as a superposition of states:

$$
\Psi_{i j \ldots k}^{\prime}=\Phi_{i}^{\mathrm{S}_{1}} \Phi_{j}^{\mathrm{S}_{2}} \ldots \Phi_{k}^{\mathrm{S}_{r}} \Psi^{\mathrm{S}_{r+1}} \ldots \Psi^{\mathrm{S}_{n}} \Psi^{\mathrm{O}}\left[\alpha_{i}^{1} \alpha_{j}^{2} \ldots \alpha_{k}^{r}\right]
$$

each of which describes the observer with a certain sequence in memory:

$$
\left[\alpha_{i}^{1} \alpha_{j}^{2} \ldots \alpha_{k}^{r}\right]
$$

Note that the state of the system with respect to the memory of the observer is a product of the system's eigenfunctions $\Phi_{i}^{\mathrm{S}_{1}}, \Phi_{j}^{\mathrm{S}_{2}}, \ldots, \Phi_{k}^{\mathrm{S}_{r}}$, whereas the remaining wave functions of the system are left the same.

In the memory of the observer, a configuration like (21) exists, which is random for an observer who fixed a certain world and nonrandom in nature. Some numbers in the sequence are the same, and some are not. Different branches will possess different sequences. 
From the above, it is concluded that the observer (who can also be an automation) shows in one of the worlds exactly what is actually shown by the apparatus measuring the quantum object. And this without any use of the wave packet collapse concept!

\subsection{Discussion topics}

Unfortunately, both the proponents and opponents of the Everett interpretation are as often as not accurate or even incorrect when interpreting his world splitting idea. It is said about the splitting of the particle and the observer into 'copies' or 'twins' as of something tantamount to their 'multiplication' at measurement. This view is inconsistent with the laws of conservation of energy and charge and is physically meaningless.

Multiplication occurs in Hilbert space; what multiplies is the states of an object, not the objects themselves.

An electron that is single before remains so after measurement (as does the observer: no twins there!) and is described at once by many relative states. The observer views one result in one of these states, and another in the other state. This means that a man in one and the same (nonmultiplying) body resides in many states at once, so that he surveys one result in one state, another in another, yet another in yet another, and so on. It is not without reason that DeWitt himself called this situation schizophrenic [16]. This is something to consult a psychiatrist on, though, who may have something useful to say to a physicist on this.

Pictures by Escher or some works painted by Salvador Dali may also serve to illustrate the splitting idea. In Escher's Wild Geese, for example, the birds look white when they fly in one direction and black when they fly in the opposite direction. So, one and the same picture can be called White-Black Geese since there are two states - and without the picture being multiplied.

By and large, the Everett interpretation cannot be blamed for violating conservation laws. It is the problems of preferred basis and probability that are the subject of criticism.

The problem of preferred basis. In the Copenhagen interpretation, if the observer chooses to measure an observable described by operator $\hat{A}$, he puts the apparatus readings into correspondence with the eigenfunctions and eigenvalues of this operator. An Everettian observer makes no choices: all that happens does so according to the Schrödinger equation. But then the wave function of the particle can be expanded on any orthonormal basis, not only in terms of the one chosen in the Copenhagen interpretation.

The answer of an Everettian uses the concept of decoherence. If the apparatus consists of a large number of particles, then a preferred basis exists which allows one, owing to decoherence, to 
speak of the approximate absence of interference between various terms in the superposition of the states of the macroobject.

It then follows that the split 'worlds' do not interact with each other, and the observer residing in one state knows nothing of the existence of other states. It is also noted that, due to the interaction of the body of the observer with other macroobjects, the states of the Universe ultimately split, making it necessary to speak of 'many universes' 65. Here, however, the same inaccuracy arises: this is not a multitude of universes but rather one Universe occupying a multitude of states. Recalling Schrödinger's cat, it can be said that he is alive in one universe and dead in another, etc.

By way of objection, though, some measurements are performed with nonmacroscopic apparatuses. An example is the Stern-Gerlach electron spin experiment, in which silver ions that play the role of an apparatus move along quasiclassical trajectories in one direction or another, and decoherence hardly plays any role.

The problem of probability. In the Everett interpretation, all events occur deterministically, and there is no random quantity which takes one or another values with some probabilities. On the other hand, as already noted above, Everett obtained a certain countably additive measure on superposition elements, which is equal to the Born rule value. What is the meaning of this measure?

One attempted answer is that an individual observer in one of the worlds, who knows nothing of the existence of other worlds, perceives herself as having appeared by accident in this world. Many of the Everettians, though - unlike Everett himself - find it necessary to resort to consciousness, which is, of course, some capitulation to Copenhagen ... .

The following example, proposed by Tegmark [65], illustrates things nicely.

A surgery patient wakes up from general anesthesia and finds herself in a ward with a definite number. If we consider general anesthesia as a quantum measurement, there are many copies (states) the patient can be in, of which she conceives only one. After another operation under general anesthesia, the patient may find herself in a ward with a different number. So the number is a random quantity for the patient. But what is the probability of identifying the observer with a fixed copy? If all the worlds are equally existent, we could assign the same probability to each of the worlds conceived by the observer, which is at odds with quantum physics. Thus, if a state is an eigenfunction of the operator of the $z$-component of electron spin, and the quantity being measured is the projection of the spin operator onto a certain axis other than normal to the $z$-axis, then the probabilities of this outcome or others are determined by the squares of the cosine and sine of the angle between above-specified axes, and these in no way equal $1 / 2$. If one tries to express 
probability in terms of the outcome frequency of a certain 'world', this frequency can be arbitrary, a point with which the Everettians themselves agree. Thus, they denounce von Mises's frequency (or statistical) interpretation of probability.

An alternative proposal put forward by Deutsch [71] is a subjective, frequency-unrelated probability interpretation used in decision making theory. It is this probability which is identified with that obtained from the Born formula.

By way of example, if you are told that, depending on your decision, you can win a thousand dollars, five hundred dollars, or nothing in three cases, then, unaware of the frequency of wins, you rely on your subjective assessments to make a decision. Of course - noting that you know nothing of any frequencies! - this requires considering a certain objective property you 'know' of, in order to avoid frustration. This can be, for example, the symmetry or perhaps some distortion in the symmetry of the coin (in the heads or tails game), or this can be the past experience of the decision-maker.

The Everett interpretation involves a multitude of worlds with an observer in them. However, the observer perceives 'herself' (or 'me') as 'embodied' in one of the worlds, whereas his other states (or 'copies', to use an Everettian term) observe (or conceive of) nothing. What is it that determines this 'embodiment' or this 'preference' of one world over another?

The only thing that features in the mathematical theory is the coefficients in the superposition or weights as their absolute values squared. This makes it tempting to consider these different coefficients as something like elements of the payment matrix in a quantum game. The larger the coefficient, the more preferred this world for the observer.

On this, Tegmark [65] says that the observer undergoing splitting will consider the Born formula as determining a certain measure on the worlds. This measure is determined based on the properties of Hilbert space. If there is initial wave function before and a different one after the measurement, nothing other than a scalar product of these two wave functions and its absolute value squared (which is similar to the absolute value squared of the coefficient in the superposition) can be proposed as such a measure. The weights will be different for various wave functions characterizing worlds. Is it possible, however, to agree with Saunders [72, 73] that, in Everett's theory, which insists on unitary evolution, the probability amplitudes of worlds are - like weights — unobservable, unlike the probability amplitude in the Copenhagen interpretation? Everett himself, judging from his paper [14], did not agree with this. According to formulas (19)-(21) of the present paper, the 'memory' of the observer stores the observation 'frequencies' of those or the other worlds. Were this otherwise, it would be completely impossible to speak of quantum mechanics and its verification 
experiments which involve, in particular, the diffraction and interference of electrons (when the frequency with which a particle hits one point or another is measured).

However, does this mean that, to use Everettian language, we survey the fingerprints of different worlds in a picture of a diffraction pattern? And to which world (or universe) does this picture now belong? Can it be said that a multitude of past worlds meet in the present one?

Furthermore, as shown by Graham [25], the frequencies that are determined, as suggested by Everett, from the number of coincident values in the memory of the apparatus [see Eqns (19) $-(21)$ ] may be arbitrary, which is precisely the reason for Saunders's statement.

Returning to Deutsch's idea on decision making theory, we note, however, that, while in decision theory as applied to business there is nothing unclear, it is impossible to understand why the observer prefers to be 'embodied' in one world and not in another, given that he will have neither benefit no matter whether the electron spin is up or down.

Another possible line of reasoning is possible here, which is similar to the one we used when discussing the Copenhagen interpretation.

According to the Bohr-Fock principle of relativity with respect to the means of measurement, augmented by Everett's notion of a relative state of a subsystem, a definite reading of the apparatus signifies a 'relation' between the particle and the observer. Adopting the 'neo-Everettian' viewpoint that the observer's consciousness is important in identifying his state with one of the split states, whereas other states exist but are not perceived by him, we could say the following. An electron, which, while possessing something like freedom of choice, is unable to make decisions because of the absence of consciousness, is characterized by many 'relations' to the observer. The Born measure can be understood as defined on these relations (characterizing, by definition, both the electron and the observer). As an electron with the same wave function is repeatedly the subject of measurements, the observer acquires a 'tendency' to find himself more often in a world with a larger Born weight. The reason is that repeated measurements of a single electron with a preassigned wave function have the consequence that, as noted above, the observer perceives himself in the same world. If the weight does not differ significantly from unity, the observer is more likely to see herself in the same place (the same world).

Tendency is a fragile thing, however, and can be denounced, and then other, even small-value, weights are realized, defined on the relations between the observer and the electron. It is the fragile nature of the tendency to identify the state of the observer with one of the worlds, which is the reason for quantum-mechanical indeterminism. But is the choice of a world something dependent on the will of the observer, as the advocates of the decision making approach claim, or is it not? 
Because the Born measure describes not only the observer but also the subject (observer)-object (particle) unity, the answer is likely to be negative.

It is not (not only!) by her will that the observer conceives herself in a fixed world. Consciousness as a choice of one of the alternatives (here we agree with Menskii [20]) or worlds should not be treated as a property of the brain or of something 'privatized' by a given observer but as a nonlocal property that embraces both the observer and the object of cognition. As the philosophy of intuitivism formulates it, "the object of knowledge is immanent in knowledge" [74].

Cognition is an objective process which, like time, embraces both the observer and the observable. Consciousness is a nonlocal property of the electron and the observer and is not related to the brain alone. The Born formula exhibits a characteristic of this nonlocal property.

Here, we should agree with DeWitt [16] that quantum probability is a special kind of probability, which does not reduce to any classical probability, in particular, to the subjective probability involved in decision making theory. The equal existence of worlds does not mean that, similar to throwing a die with six identical faces, the observer can adopt the classical definition of probability and assign the same probability to all worlds. Is this answer convincing? Hardly. All this reasoning about an observer, decisions making by her, and consciousness etc. have no relation to the Schrödinger equation and quantum-mechanical mathematics (thought of at the very start as all-describing) and do nothing more than revive the old wave packet collapse debate - but in a much more vague and nebulous manner this time.

The role of consciousness in the Everett interpretation is also discussed by Brandon Carter [64, who rejects Deutsch's decision making approach.

The dilemma covering the equal existence of different worlds in a superposition and the different probabilities of their observation according to Born's measure is proposed to be resolved by analogy with the Russian roulette game, an invention of Russian white-guard officers, in which a revolver is loaded with (say) four blanks and one bullet. From the point of view of outcomes, the probability of some outcome is one in five, but from the point of view of a potential suicide, the probability of remaining alive is four in five, and of dying, one in five.

Of importance in this context is the identification and distinguishing of worlds. The observer identifying herself with one of the copies can identify some worlds and introduce different weights for the group of worlds she identifies and for those she distinguishes. It remains unclear, though, how to obtain exactly the Born formula, and why, in fact, this should be the Born formula, and why it should be obtained at all, for that matter. The rules of quantum physics are unlikely to be derived from any classical analogs whatsoever. 
Carter also levels criticism at the concept of 'splitting worlds' by arguing that worlds 'exist eternally' and that all there is to talk about is distinguishing and identifying them. Clearly, the Schrödinger equation should be applied to all (an infinite number of) wave functions, which is reminiscent of Feynman's critical remark that the Everett theory loses much of its validity because of the necessity to consider an infinite number of probability amplitudes. Criticisms of, and indeed accusations of inconsistency against, the subjective prob- ability approach come from Kent [75]. In any case, the present author's view is that this approach, while interesting, is not sufficiently grounded to enter quantum physics textbooks.

Thus, we should agree with Kent [75] that Everettians are often contradictory in their publications and have as yet no consensus on how the Everett interpretation should be interpreted.

Some authors, for example DeWitt, emphasize that consciousness does not play any role, while others argue the opposite and try to employ decision making theory. Finally, Deutsch [63] himself, the chief pro-Everettian, notes, quite rightly, that such aspects as the smearing out of the wave packet, a situation where worlds obviously interact and where interference is involved, have not been given sufficient analysis. Even the highly popular two-slit experiment with a quantum particle has not been treated this way.

Opinions differ not only on the 'splitting' of worlds but also on their 'merging' (see above). Some agree that merging is necessary [65] and some do not, because they do not consider the merger of macroscopic decoherent worlds possible.

\section{Conclusion}

To summarize, our preference goes, of course, to the Copenhagen interpretation, because it is worked out more and agrees with all observations in the microworld. Materialistic prejudices that determine the rejection and prevent adoption of the Copenhagen interpretation by some physicists can hardly be considered as a serious argument [76]. Unfortunately, strengthening the case of materialism in the eyes of many scientists is their desire to distance themselves from various kinds of parascience that discredits nonmaterialistic philosophy.

It is, however, curious - and bitterly ironic - that anti-Copenhagenists themselves, with their self-proclaimed 'realistic' many-worlds interpretation, find themselves obliged to speak of consciousness and of the necessity to identify the observer with something fixed in a certain world, thereby demonstrating, in a sense, the convergence of above interpretation. We put aside, of course, the wild talk about Everettian parallel-world mysticism typical of fantasy fiction, cinema, and mass 
media 69].

But also in the Copenhagen interpretation itself, if it is also considered realistic, with observerindependent quantum reality described by operators and not by numbers (as explained by Aage Bohr), time evolution should, as mentioned above, be described in the Heisenberg representation. Thus, quantum reality in the language of operators contains - due to the possibility of spectral decomposition in projectors into the eigenstates - not a single world but many worlds at once, and evolution is the evolution of all these worlds simultaneously. This is, of course, very close to Everett's picture.

Still, the Copenhagen interpretation, with its emphasis on the role of consciousness, may have something new to say about the relation between consciousness and the brain. This, according to von Neumann, requires drawing a boundary between the two. The thirty plus years of discussions conducted by the present author at the Institute of Human Brain of the Russian Academy of Sciences (St. Petersburg), while not producing any definite results, have established a number of points of agreement.

(1) Brain specialists have become interested in the nonlocality, or entanglement, of quantum states of many particles - properties that have analogs in the nonlocal aspects of the operation of the brain as a whole.

(2) The main question common to philosophy, psychology, and brain theory is formulated as a psychophysical mental issue. How does it happen that obviously nonmaterial mental processes control and affect the physical and chemical processes in the brain and the body? A possible answer [77] can be hidden in quantum physics with its negative experiments in which, while negative information involves no energy or momentum transfer from the observer (apparatus) to the quantum object being observed, it nevertheless changes the energy and momentum of the object (to a degree allowed by the uncertainty relation). Thus, nonmaterial consciousness, which does not possess physical energy and momentum, can lead, due to its main property (consisting in 'cognition of information'), to physical and chemical consequences.

(3) What is unique about my conceiving of processes in my body is that consciousness considered as a quantum apparatus is not material — like the mental in general — and, hence, does not consist of quantum particles, and, further, cannot be thought of in terms of decoherence.

Information on the physical characteristics of the body is formulated not in physical terms like the position of the pointer in a measuring instrument but in purely mental terms like joy, pain, and feelings like these. That this 'apparatus' possesses no ability to decoherence means that, unlike an ordinary apparatus used in physical experiment, it can also observe other than classical 
observables... I can observe Schrödinger cats in my body. Curiously, any external observation of the brain using physical apparatuses in some way or another prevents me, due to the decoherence that operates in this case, from surveying this interaction between my consciousness and my body. It is well known, though, that you can cut the brain in whatever way but you will never watch consciousness. 'Quantum conspiracy' seems to be an appropriate term here.

A major difficulty in discussions with biologists and medical people is their traditional materialistic position determinate mechanistically in the spirit of Newton, a position which is less characteristic of physicists. There have been two definite exceptions, though: N P Bekhtereva, a person of great freedom of thought, always interested in the philosophical assessment of the results of her long-running brain studies, and S V Medvedev, Director of the Institute of Human Brain and a physics graduate.

To conclude, our view is that, while the many-world interpretation is currently not sufficiently elaborated and is rather a compilation of less than convincing and sometimes poorly connected ideas, the current understanding that most of the Universe is occupied by unobservable (invisible) dark matter and dark energy calls for more serious attention to a theory in which the existence of many unobserved worlds is assumed as an axiom. The question is how these worlds gravitate.

\section{Acknowledgments}

This work was carried out in cooperation with the Copernicus Center for Interdisciplinary Studies in Kraków, Poland, under financial support from the Templeton Foundation.

\section{References}

[1] Grib A A, Petrova D V Russ. Phys. J. 53803 (2011) [Izv. Vyssh. Uchebn. Zaved. Fiz. (8) 32 (2010)]

[2] Kochen S, Specker E P J. Math. Mech. 1759 (1967)

[3] Cassinello A, Gallego A Am. J. Phys. 73273 (2005)

[4] Belinskii A V Phys. Usp. 40305 (1997) [Usp. Fiz. Nauk 167323 (1997)]

[5] Belinskii A V Phys. Usp. 37219 (1994) [Usp. Fiz. Nauk 164231 (1994)]

[6] Belinskii A V Phys. Usp. 37413 (1994) [Usp. Fiz. Nauk 164435 (1994)]

[7] Aspect A, Dalibard J, Roger G Phys. Rev. Lett. 491804 (1982)

[8] Bouwmeester D et al. Nature 390575 (1997) 
[9] Bohm D, Hiley B J The Undivided Universe: an Ontological Interpretation of Quantum Theory (London: Routledge, 1993)

[10] Wigner E P, in The Scientist Speculates (Ed. I J Good) (London: Heinemann, 1961) p. 284

[11] Gell-Mann M, Hartle J B Phys. Rev. D 473345 (1993)

[12] Kotel'nikov V A Phys. Usp. 52185 (2009) [Usp. Fiz. Nauk 179204 (2009)]

[13] Bohr A, Ulfbeck O Rev. Mod. Phys. 671 (1995)

[14] Everett H (III) Rev. Mod. Phys. 29454 (1957); in The Many-Worlds Interpretation of Quantum Mechanics (Eds B S DeWitt, N Graham) (Princeton, N.J.: Princeton Univ. Press, 1973) p. 141

[15] Wheeler J A Rev. Mod. Phys. 29463 (1957); in The Many-Worlds Interpretation of Quantum Mechanics (Eds B S DeWitt, N Graham) (Princeton, N.J.: Princeton Univ. Press, 1973) p. 151

[16] DeWitt B, in The Many-Worlds Interpretation of Quantum Mechanics (Eds B S DeWitt, N Graham) (Princeton, N.J.: Princeton Univ. Press, 1973) p. 167

[17] Hartle J B, Hawking S W Phys. Rev. D 282960 (1983)

[18] Hawking S W A Brief History of Time: from the Big Bang to Black Holes (Toronto: Bantam Books, 1988) [Translated into Russian (St. Petersburg: Amfora, 2007)]

[19] Vilenkin A Many Worlds in One: the Search for Other Universes (New York: Hill and Wang, 2006) [Translated into Russian (Moscow: Astrel', 2010)]

[20] Menskii M B Phys. Usp. 43585 (2000) [Usp. Fiz. Nauk 170631 (2000)]

[21] Menskii M B Phys. Usp. 48389 (2005) [Usp. Fiz. Nauk 175413 (2005)]

[22] Kadomtsev B B, Kadomtsev M B Phys. Usp. 39609 (1996) [Usp. Fiz. Nauk 166651 (1996)]

[23] Lesovik G B Phys. Usp. 44429 (2001) [Usp. Fiz. Nauk 171449 (2001)]

[24] von Neumann J Mathematische Grundlagen der Quantenmechanik (Berlin: J. Springer, 1932) [Translated into English: Mathematical Foundations of Quantum Mechanics (Princeton, N.J.: Princeton Univ. Press, 1955); translated into Russian (Moscow: Nauka, 1964)]

[25] Graham N, in The Many-Worlds Interpretation of Quantum Mechanics (Eds B S DeWitt, N Graham) (Princeton, N.J.: Princeton Univ. Press, 1973) p. 229

[26] Hartle J B Am. J. Phys. 36704 (1968)

[27] Fock V A Kvantovaya Fizika i Stroenie Materii (Quantum Physics and the Structure of Matter) (Moscow: Librokom, 2010) 
[28] Grib A A, Rodrigues W A (Jr.) Nonlocality in Quantum Physics (New York: Kluwer Academic/Plenum Publ., 1999)

[29] Grib A A Sov. Phys. Usp. 27284 (1984) Usp. Fiz. Nauk 142619 (1984)]

[30] Grib A A Narushenie Neravenstv Bella i Problema Izmereniya v Kvantovoi Teorii (Violation of the Bell Inequality and the Problem of Measurement in Quantum Theory) (Lectures for Young Scientists, P2-92-211) (Dubna: JINR, 1992)

[31] Vladimirov Yu S Osnovaniya Fiziki (Foundations of Physics) (Moscow: Binom. Lab. Znanii, 2008)

[32] Shimony A, in Penrose R et al. The Large, the Small, and the Human Mind (Cambridge: Cambridge Univ. Press, 1999) [Translated into Russian: in Hawking S et al. Bol'shoe, Maloe i Chelovecheskii Razum: Spor o Fizicheskom Mire i Mire Idei (St. Petersburg: Amfora, 2012) p. 14]

[33] Heisenberg W Physik und Philosophie (Stuttgart: S. Hirzel, 1959) [Translated into English: Physics and Philosophy (Amherst, N.Y.: Prometheus Books, 1999); translated into Russian (Moscow: Nauka, 1989)]

[34] Finkelstein D Quantum Relativity: a Synthesis of the Ideas of Einstein and Heisenberg (Berlin: Springer, 1996)

[35] Grib A A, Mamaev S G, Mostepanenko V M Vakuumnye Kvantovye Effekty v Sil'nykh Polyakh (Vacuum Quantum Effects in Strong Fields ) (Moscow: Energoatomizdat, 1988)

[36] Grib A A Vestn. Leningrad. Gos. Univ. (10) 61 (1969)

[37] Everett H (III), in The Many-Worlds Interpretation of Quantum Mechanics (Eds B S DeWitt, N Graham) (Princeton, N.J.: Princeton Univ. Press, 1973) p. 3

[38] Schopenhauer A Die Welt als Wille und Vorstellung (Leipzig: F. A. Brockhaus, 1859) [Translated into English: The World as Will and Presentation (New York: Pearson Longman, 2008 - 2011); translated into Russian (Moscow: Prosveshchenie, 1990)]

[39] Schrödinger E What is Life? The Physical Aspect of the Living Cell (Cambridge: The Univ. Press, 1945) [Translated into Russian (Moscow: IL, 1947)]

[40] London F, Bauer E La Théory de l'observation en Mécanique Quantique (Paris: Hermann and Cie, 1939)

[41] Wigner E P, in The Scientist Speculates (Ed. I J Good) (London: Heinemann, 1961) p. 204 
[42] Penrose R The Emperor's New Mind: Concerning Computers, Minds, and the Laws of Physics (New York: Penguin Books, 1991) [Translated into Russian (Moscow: URSS, 2003)]

[43] Squires E Conscious Mind in the Physical World (Bristol: A. Hilger, 1990)

[44] Menskii M B Phys. Usp. 44438 (2001) [Usp. Fiz. Nauk 171459 (2001)]

[45] Menskii M B Phys. Usp. 50397 (2007) [Usp. Fiz. Nauk 177415 (2007)]

[46] Penrose R, in Penrose $\mathrm{R}$ et al. The Large, the Small, and the Human Mind (Cambridge: Cambridge Univ. Press, 1999) [Translated into Russian: in Hawking S et al. Bol'shoe, Maloe i Chelovecheskii Razum: Spor o Fizicheskom Mire i Mire Idei (St. Petersburg: Amfora, 2012) p. 58]

[47] Renninger M Z. Phys. 158417 (1960)

[48] Birkhoff G, von Neumann J Ann. Math. 37823 (1936)

[49] Jauch J M Foundations of Quantum Mechanics (Reading, Mass.: Addison-Wesley Publ. Co., 1968)

[50] Piron C, Ph.D. Thesis (Lausanne: Univ. Lausanne, 1993)

[51] Grib A A Int. J. Theor. Phys. 322389 (1993)

[52] Hepp K Helv. Phys. Acta 452237 (1972)

[53] Menskii M B Phys. Usp. 461163 (2003) [Usp. Fiz. Nauk 1731199 (2003)]

[54] Bell J S Speakable and Unspeakable in Quantum Mechanics (Cambridge: Cambridge Univ. Press, 1987) p. 117

[55] Zurek W H Phys. Today 44 (10) 36 (1991)

[56] Ivanitskii G R Phys. Usp. 53327 (2010) [Usp. Fiz. Nauk 180337 (2010)]

[57] Reutov V P, Schechter A N Phys. Usp. 53377 (2010) [Usp. Fiz. Nauk 180393 (2010)]

[58] Grib A A Metodologicheskoe Znachenie Kvantovoi Fiziki dlya Psikhologii (Pamyati V.A. Foka) [Methodological Implications of Quantum Theory for Psychology (In memory of V A Fock)] (Leningrad: Izd. LGU, 1980)

[59] Shikhovtsev E B NeuroQuantology 11 (Suppl. 1) 160 (2013)

[60] Byrne P Sci. Am. 297 (6) 98 (2007)

[61] Schrödinger E The Interpretation of Quantum Mechanics: Dublin Seminars (1949 - 1955) and Other Unpublished Essays (Woodbridge, Conn.: Ox Bow Press, 1995) 
[62] Byrne P, in Many Worlds? Everett, Quantum Theory, and Reality (Eds S Saunders et al.) (Oxford: Oxford Univ. Press, 2010) p. 521

[63] Deutsch D, in Many Worlds? Everett, Quantum Theory, and Reality (Eds S Saunders et al.) (Oxford: Oxford Univ. Press, 2010) p. 542

[64] Carter B, arXiv:1203.0952

[65] Tegmark M, in Many Worlds? Everett, Quantum Theory, and Reality (Eds S Saunders et al.) (Oxford: Oxford Univ. Press, 2010) p. 553

[66] Page D N Int. J. Mod. Phys. D 5583 (1996)

[67] Vaidman L "Many-Worlds interpretation of quantum mechanics", The Stanford Encyclopedia of Philosophy, Summer 2002 ed. (Principal Ed. E N Zalta) (Stanford: Stanford Univ., 2002); http:// plato.stanford.edu/archives/sum2002/entries/qm-manyworlds/

[68] Tegmark M Nature 44823 (2007)

[69] Deutsch D Proc. R. Soc. Lond. A 4553129 (1999)

[70] Kaku M Physics of the Impossible (New York: Doubleday, 2008) [Translated into Russian (Moscow: Dinastiya, 2012)]

[71] Deutsch D The Fabric of Reality: the Science of Parallel Universes and Its Implications (New York: Allen Lane, 1997)

[72] Saunders S, in Many Worlds? Everett, Quantum Theory, and Reality (Eds S Saunders et al.) (Oxford: Oxford Univ. Press, 2010) p. 3

[73] Saunders S, in Many Worlds? Everett, Quantum Theory, and Reality (Eds S Saunders et al.) (Oxford: Oxford Univ. Press, 2010) p. 151

[74] Losskii N O Obosnovanie Intuitivizma (The Foundations of Intuiti- vism) (Notes of Department of History and Philology of Imperial St. Petersburg Univ., Ch. 78) (St. Petersburg: M.M. Stasyulevich Printing House, 1906)

[75] Kent A, in Many Worlds? Everett, Quantum Theory, and Reality (Eds S Saunders et al.) (Oxford: Oxford Univ. Press, 2010) p. 307

[76] Popov M A Phys. Usp. 461307 (2003) [Usp. Fiz. Nauk 1731382 (2003)]

[77] Grib A A, Revista Electronica de Letras, RP 11/99 (Brasil: IMECC, UNICAMP, 1999) 